\title{
Way Forward: Gastrointestinal Tract Endoscopy Work Flow Postlockdown Era
}

\author{
Randhir Sud ${ }^{1}$ Sukrit Sud ${ }^{1}$ \\ ${ }^{1}$ Institute of Digestive and Hepato-biliary Sciences, Medanta the \\ Medicity, Gurgaon, Haryana, India
}

J Digest Endosc 2020;11:89-91

\begin{abstract}
Address for correspondence Randhir Sud, MD, DM, Institute of Digestive and Hepato-biliary Sciences, Medanta the Medicity, Sector 38, Gurgaon 12200, Haryana, India (e-mail:drrsud@gmail.com).
\end{abstract}

\begin{abstract}
Keywords

- severe acute respiratory syndrome coronavirus 2

- health care workers

- endoscopy

- colonoscopy

- aerosol

Gastrointestinal tract endoscopy being an aerosol generating procedure increases the risk to staff and uninfected patients from a coronavirus disease 2019 patient. Social and physical distancing through "lockdown" has suppressed the spread of disease but will not eradicate it. Various endoscopy societies formulated guidelines to triage the patients and limit the work to only emergency and urgent cases and postpone "routine" endoscopies. Postlockdown infected vector pool will persist till an effective vaccine is widely available. Nonurgent cases cannot be postponed indefinitely. We need to identify infected symptomatic and asymptomatic individuals and create a safe environment for uninfected patients. Endoscopy staff protection through education, optimized manpower flow, and personal protective equipment usage and hand hygiene needs urgent attention. Proper environment sanitization, endoscope, and device reprocessing will remain important.
\end{abstract}

We are living through unprecedented times faced with a pandemic due to a zoonotic virus, which acquired human to human spread. Severe acute respiratory syndrome coronavirus 2 (SARS-CoV-2) virus spreads through exposure to droplets in aerosol of an infected person directly or through infected surfaces contaminated by these secretions. ${ }^{1}$ Virus may remain viable for up to 3 days in aerosol and up to few days on various surfaces. Viral RNA is also detected in stool even after pharyngeal swabs have cleared, ${ }^{2}$ though feco-oral transmission and infectivity of feces and aerosol during flatus is not proven. SARS-CoV-2 is highly infectious and carries significant mortality in vulnerable population. Oral endoscopic procedures, like upper gastrointestinal tract endoscopy, endoscopic retrograde cholangiopancreatography, and endoscopic ultrasound, are aerosol generating procedures and because of fecal viral shedding lower GI endoscopic procedures also remain potentially infective.

Health care workers (HCWs) by very nature of their job are more susceptible to this infection. In Italy more than $20 \%$ HCW got infected with SARS-CoV-2. ${ }^{3}$ In United States, nearly 9,300 HCWs were infected by April 12, $2020 .{ }^{4}$ Endoscopy staff involved in pre-endoscopy evaluation and in the procedures is at risk of infection from a coronavirus disease 2019 (COVID-19) patient or asymptomatic vector. They, in turn, can be the source of infection to susceptible patients and contacts including their family.

During the pandemic, our main aim is to contain the virus so that the number of people needing hospitalization or ICU and ventilatory care do not overwhelm our meager health care resources. Protection of HCW is an equally important goal at this point as they are a valuable resource to take care of sick and needy. To reduce human to human spread, major strategy used by many countries including India is to minimize physical interaction by "lockdown." At present nearly $1 / 3$ of the world population is under lockdown including 1.35 billion people in India. During this period, various endoscopy societies issued guidelines ${ }^{5}$ with the primary aim of protecting the endoscopy staff and non-COVID-19 patients from acquiring the infection. It was advised to restrict the endoscopy procedures to emergency and semiurgent indications only and postponing elective endoscopies for few weeks. ${ }^{6}$ This is being followed by most units in India, resulting in endoscopy volumes falling to 10 to $20 \%$ of usual load and many in standalone GI practice have stopped doing endoscopies totally. 
Lockdown in India seems to have prevented catastrophic escalation of infection, but it comes at a huge social and economic cost. It has to end sooner or later. SARS-CoV-2 will not disappear by then. It will persist either as a protracted pandemic or at a lower level with periodic recrudescence till one of the following happens:

a) Herd immunity develops in $>50 \%$ population and number of susceptible populations keeps decreasing. It is estimated that nearly $40 \%$ population in Spain and $26 \%$ in Italy has been infected and may have acquired immunity. In India, we are nowhere near it.

b) Effective vaccine is developed and delivered. This is at least 2 years away.

c) Very effective antiviral therapy evolves reducing the number of vectors overtime like HCV infection.

None of these is happening anytime soon. We need to restrategize our endoscopy workflow after the lockdown is lifted. As indeterminate number of asymptomatic and symptomatic COVID-19 patients will be present among the outpatients department, in-patients, and endoscopy patients, we need to have stringent safeguards in place to keep nonCOVID-19 patients, endoscopy staff, and their contacts safe.

Nonurgent endoscopies cannot be postponed indefinitely, and we have to move from "let us not do them" to "let us do them safely."

There are no published guidelines or studies for us to follow. We have to move from our highly restrictive approach about indications of endoscopy to more liberal but cautious approach without compromising safety. Following suggestions are made based on scanty published literature and expert opinions.

\section{Patient Prescreening for Endoscopy}

Asian Pacific Society for Digestive Endoscopy position statement and Society of Gastrointestinal Endoscopy of India-COVID-19 statement suggest patient triaging according to clinical history. Patients are asked about fever $>37.5^{\circ} \mathrm{C}$ (F), h/o travel to places with high prevalence (T), occupation to assess potential for infection ( 0 ), contact with a case of COVID-19 or traveler in last 14 days (C), place of stay (cluster or hot spot)-fever, travel, occupation, contact, and clustering. Any one of these positive should be tested for SARS-CoV-2.

This history and symptoms-based screening will be of limited value as the pandemic becomes protracted and community spread happens. Median time for an infected person to become symptomatic is 5 days and could be up to 11 days and up to $80 \%$ are asymptomatic or mildly symptomatic but still infective ${ }^{1,7}$ without any $\mathrm{h} / \mathrm{o}$ travel or obvious contact. Such patients may come for endoscopy. For next 3 months, we can assume persistence of the pandemic and community spread at a variable rate.

Endoscopy guidelines for emergency, semiurgent endoscopy, endoscopy in COVID-19 positive, and COVID probable cases are already defined, and we should continue to follow these. $^{5}$
For routine endoscopy, testing for virus by doing single or serial real-time polymerase chain reaction (RT-PCR) from pharyngeal swabs 2 weeks apart before routine endoscopy will be justified ${ }^{8}$ If the incidence in a particular region is low, then pooled RT-PCR for three to five patients may be more cost-effective strategy for screening. Use of reliable antibody test when available may be a cheaper and quicker alternative. A negative test reassures you of a lack of contact with the virus. Immunoglobulin $\mathrm{M}(\operatorname{IgM})$ antibody titers develop in 50 to $80 \%$ in 1 week and immunoglobulin G in 90 to $100 \%$ after 2 to 4 weeks. ${ }^{9}$ With the available evidence a clinically low-risk patient with negative antibody test and IgG positive patient can be assumed virus free while a IgM positive patient should have pharyngeal swab for RT-PCR to rule out active infection.

Every patient should be advised to don a face mask and temperature checked before coming to endoscopy area.

Evaluation of the patient and consenting shall be done with proper protection and hand hygiene. Endoscopy holding area should maintain appropriate distance between patients and sanitize appropriately for preventing fomite-related transmission.

\section{Endoscopy Facility and Staff Use}

Patient apprehension and difficulty in commuting will only improve slowly and so will flow of nonurgent endoscopy. We should plan to run our facilities at 50\% capacity for next 3 months and change it afterwards according to the prevailing situation. Office endoscopy with less secure environment may not be possible for quite some time.

Some guidelines suggest doing proven or suspected COVID-19 patients in negative pressure room to avoid persistence of aerosol. ${ }^{10}$ If available, we should use such facility also for emergency endoscopy when wait for the test is not feasible. In case this pandemic truly drags on, endoscopy centers may have to create such a facility.

Restricting the staff in endoscopy suite to one endoscopist, one technician, and one nurse will ensure optimal personal protective equipment (PPE) usage and minimize risk of exposure to the staff. Endoscopy staff should preferably be divided into two teams working on alternate days. This avoids shut down of endoscopy unit if the staff gets compromised on a particular day. Another strategy is weekly rotation of endoscopy staff. Half of them on duty and other half at rest. Advantage being if the staff at rest remains symptom free then may can be assumed to be uninfected. Age and comorbidity are risk factors for serious COVID-19 disease and mortality. It may be prudent that high-risk staff ( $>60$ years age, diabetes, hypertension, or immune compromised) should avoid exposure during the protracted pandemic.

\section{Personal Safety Steps}

Appropriate PPE for the endoscopy staff ${ }^{11}$ including hairnet, FFP2/3 or N 95 face mask, face shield or goggles, water resistant gown, shoe covers and gloves must be mandatory. 
Every endoscopy room should have contactless hand sanitizer dispenser for liberal use.

Same doctor and nurse team should do preprocedure evaluation, the procedure and postprocedure monitoring to limit exposure of minimal staff to every patient. The technician assisting the procedure should complete the scope reprocessing.

Once oxygen saturation is $>90 \%$ face mask should be put on the patient to reduce cough-related droplet spray. ${ }^{12}$

Viral RNA is detectable in feces, but infectivity is still not established. We should still observe the same safety protocol during colonoscopy as for oral procedures.

Doing the procedures under deep sedation reduces aerosol generation and possibly reduces risk to the staff. Using continuous suction while doing accessory exchange through biopsy port is another possible strategy to reduce contamination. ${ }^{13}$

Endoscopy room should be as bare as possible to minimize fomite contamination and no exposed nonessential material should be around. Strict endoscopy suite sanitation with sodium hypochlorite after each procedure and thorough sanitation of all the equipment at the end of work should become routine.

\section{Chemoprophylaxis of Health Care Workers}

At present, there is no scientific evidence of effective pre-exposure prophylaxis with any drug including hydroxychloroquine. There is one unpublished trial of recombinant human interferon $\alpha$ nasal drops with or without thymosin injection in 2,944 doctors and nurses in Hubei province China with zero infection rate in 28 days, while 2,035 unprotected HCW developed infection in the same region. ${ }^{14}$

\section{Endoscopy Staff Training and Education}

We have to prepare ourselves for a long battle and change our behavior in the endoscopy area. We need to educate and train our doctors, technicians, nursing staff, ward boys and sanitary staff about social practices, safe handling of patients, proper hand sanitation, and donning and doffing of PPE. Staff involved in endoscope and device reprocessing has to be particularly vigilant and appropriately trained.

Patient education is equally essential to make them understand the new algorithm with increased cost for a "routine" endoscopic procedure. Screening colonoscopy, surveillance colonoscopy for inflammatory bowel disease, surveillance endoscopy for Barrett's and oesophageal varices may be postponed till the pandemic is over.

Consider every patient to be potentially infected and adhere to safety protocols to keep our staff and patients safe without getting overwhelmed by the present scenario.

\section{Conflict of Interest}

None declared.

\section{References}

1 Wang C, Horby PW, Hayden FG, Gao GF. A novel coronavirus outbreak of global health concern. Lancet 2020;395(10223) :470-473

2 Chen L, Lou J, Bai Y, Wang M. COVID-19 disease with positive fecal and negative pharyngeal and sputum viral tests. Am J Gastroenterol 2020

3 Remuzzi A, Remuzzi G. COVID-19 and Italy: what next. ?Lancet 2020;395(10231) :1225-1228

4 Characteristics of Health Care Personnel with COVID-19United States. CDC Weekly Morbid Mortal Rep 2020 Available at: https://www.cdc.gov/mmwr/volumes/69/wr/mm6915e6. html Accessed April 29, 2020

5 Chiu PWY, Ng SC, Inoue H, et al. Practice of endoscopy during COVID-19 pandemic: position statements of the Asian Pacific Society for Digestive Endoscopy (APSDE-COVID statements) Gut 2020;gutjnl-2020-321185

6 Mathew P, Lakhtakia S, Agarwal R, et al. Joint Guidance from SGEI, ISG and INASL for Gastroenterologists and Gastrointestinal Endoscopists on the Prevention, Care and Management of patients with COVID-19. J Clin Experiment Hepatol 2020;doi.org/10.1016/j.jceh.2020.04.001

7 Chan JF, Yuan S, Kok KH, et al. A familial cluster of pneumonia associated with the 2019 novel coronavirus indicating person-to-person transmission: a study of a family cluster. Lancet 2020;395(10223):514-523

8 OngJ, CrossGB, DanYYThe prevention of nosocomial SARSCoV2 transmission in endoscopy: a systematic review of recommendations within gastroenterology to identify best practice.Medrxiv2020 Available at https://www.medrxiv.org/ content/10.1101/2020.03.17.20037564v1 Accessed April 29, 2020

9 Lou B, Li T, Zheng S, Su Y, Li Z. Serology characteristics of SARSCoV- 2 infection since the exposure and post symptoms onset. Norwegian Inst Publ Health 2020

10 Calderwood AH, Day LW, Muthusamy VR, et al; ASGE Quality Assurance in Endoscopy Committee. ASGE guideline for infection control during GI endoscopy. Gastrointest Endosc 2018;87(5):1167-1179

11 Sultan S, Lim JK, Altayar O, et al; AGA. AGA Institute Rapid Recommendations for gastrointestinal procedures during the COVID-19 pandemic. Gastroenterology 2020; S0016-5085(20)30458-3

12 Repici A, Maselli R, Colombo M, et al. Coronavirus (COVID-19) outbreak: what the department of endoscopy should know. Gastrointest Endosc 2020;S0016-5107(20)30245-5

13 Vavricka SR, Tutuian R, Imhof A, et al. Air suctioning during colon biopsy forceps removal reduces bacterial air contamination in the endoscopy suite. Endoscopy 2010;42(9):736-741

14 Zhongji M, Tongyu W, Li C. An experimental trial of recombinant human interferon alpha nasal drops to prevent coron:avirus disease 2019 in medical staff in an epidemic areaMedRxiv2020 Available at https://www.medrxiv.org/content/10.1101/2020. 04.11.20061473v1 Accessed April 29, 2020 